\title{
Web Engineering - Old Wine in New Bottles?
}

\author{
Gerti Kappel ${ }^{1}$, Elke Michlmayr ${ }^{2}$, Birgit Pröll ${ }^{3}$, \\ Siegfried Reich ${ }^{4}$, and Werner Retschitzegger ${ }^{5}$ \\ ${ }^{1}$ Business Informatics Group (BIG), Vienna University of Technology \\ Favoritenstr. 9, A-1040 Vienna, Austria \\ gerti@big.tuwien.ac.at \\ ${ }^{2}$ Women's Postgraduate College on Internet Technologies (WIT) \\ Vienna University of Technology \\ Favoritenstr. 9, A-1040 Vienna, Austria \\ michlmayr@wit.tuwien.ac.at \\ ${ }^{3}$ Institute for Applied Knowledge Processing (FAW) \\ Softwarepark Hagenberg, Hauptstraße 119, A-4232 Hagenberg, Austria \\ birgitefaw.uni-linz.ac.at \\ ${ }^{4}$ Salzburg Research \\ Jakob Haringer Straße 5/III, A-5020 Salzburg, Austria \\ siegfried.reich@salzburgresearch.at \\ ${ }^{5}$ Department of Information Systems (IFS), Johannes Kepler University Linz \\ Altenbergerstr. 69, A-4040 Linz, Austria \\ werneraifs.uni-linz.ac.at
}

\begin{abstract}
Modern Web applications are full-fledged, complex software systems. Therefore, the development of Web applications requires a methodologically sound engineering approach called Web Engineering. It is not clear, however, to which extent existing solutions from relevant areas, most notably software engineering can be reused as such for the development of Web applications and consequently, if Web Engineering is really a discipline on its own. This paper highlights the characteristics of Web application development as found in existing literature thus providing a prerequisite for analyzing the appropriateness of existing engineering solutions. The characteristics are categorized according to four dimensions, comprising the software product itself, its development, its use and evolution as a cross-cutting concern.
\end{abstract}

\section{Introduction}

The World Wide Web has a massive and permanent influence on our lives. Economy, industry, education, healthcare, public administration, entertainment - there is hardly a part in our daily life that has not been pervaded by the World Wide Web. The reason for this omnipresence lies especially in the very nature of the Web, which is shaped by the global and permanent availability and comfortable and uniform access to often widely distributed information producible by anyone in the form of Web pages 
$[2,25]$. Web applications today are full-fledged, complex software systems providing interactive, data intensive and customisable services accessible through different devices, working state-based for the realization of user transactions and usually storing the used data in an underlying database. Despite the fundamental changes in the orientation of the Web, from an informational to an application medium, the development of Web applications is still seen as a one-time event, spontaneous, usually based on the knowledge, experiences and development practices of individual developers, limited to be reused in the sense of the "Copy\&Paste paradigm", and ultimately characterized by inadequate documentation of design decisions. A survey of the practice of Web application development reported that there is only limited use of design techniques, documentations are produced seldom and testing procedures are most often not formalized [31]. Keeping this practice in mind, it is no surprise that a survey done by the Cutter Consortium [7] found out that the top problem areas of large-scale Web application projects were the failure to meet business needs $(84 \%)$, project schedule delays $(79 \%)$, budget overrun (63\%), lack of required functionality $(53 \%)$, and poor quality of deliverables (52\%). The current situation of ad hoc development of Web applications reminds us of the software development practices of the 1960s, before it was realized that the development of applications required more than programming expertise $[8,9,28]$.

Now, the problems seem to be the same, so are the solutions the same, too? Is the notion of Web Engineering just a new application domain of software engineering reusing and slightly adapting already existing approaches in terms of methodologies, principles, standards and best practice guides, or is it really a discipline in its own calling for new solutions (cf., e.g., $[13,15,16])$ ? One of the prerequisites to answer this question is to clarify, how far Web application development is different to traditional software development. Answering this question would allow to reason about the applicability and appropriateness of approaches already existing in relevant computing fields.

\section{Characteristics of Web Application Development}

Web applications are software systems based on technologies and standards of the World Wide Web Consortium (W3C). They provide Web-specific resources such as content and services through a user interface, the Web browser. With this definition of Web applications in mind, we try to explore the characteristics of Web application development, heavily relying on existing literature $[1,5,11,15,16,20-29,32]$. We structure our discussion into four different dimensions, comprising the software product itself, its development, its use and evolution as a cross-cutting concern [14, 19]. It has to be emphasized that we don't claim that each of these characteristics is unique to Web application development never occurring when developing traditional, i.e., non-Web applications. Characteristics mentioned in existing literature to be

1 Note that the term "ad hoc" is interpreted as "doing something in an unstructured way", and not in its original sense of "focused at the problem at hand" (cf. [12]). 
unique for Web development seem to be not that outstanding compared to certain traditional software development domains $[15,16]$. This is not least due to the fact that for each characteristic the degree of difference depends on the category of Web application considered (examples for such categorizations can be found in $[19,26$, 29]).

\subsection{Application-Related Characteristics}

When developing Web applications one has to consider not only functionality but equally address content, hypertext, and presentation aspects.

Content. The origin of the Web is its role as information medium. Beyond the required functionality, Web applications are thus heavily content-driven. Content comprises not only structured data residing in database systems but also unstructured and semi-structured data such as textual descriptions or multi-media information. Complexity arises especially from the fact that content is often highly dynamic and continuously updated. Also, users typically demand high content quality in terms of topicality, accurateness, consistency, and reliability [30]. Consequently, the development of Web applications is not only a complex engineering task but relies heavily on authors responsible for the content.

Hypertext. Web applications advocate the hypertext paradigm [6] as the fundamental paradigm for structuring information. The basic elements of the Web's notion of hypertext are nodes, links and anchors. Typical examples of accessing hypertextual information include browsing (like in online stores' catalogues), querying (like in elearning applications), or guided tours (like in virtual exhibitions). The essential feature of the hypertext paradigm is its non-linearity requiring from both authors and users to address the potential issues of disorientation and cognitive overload. This can be achieved for example through specific navigation design (site maps, keyword searches, traversed paths, etc.) and is essential to preserve quality of access [10].

Presentation. In conventional software systems the "look and feel" is often to a large extent determined by standardized user interface elements and style guides. Presentation is a central quality factor for Web applications not least to the high competitive pressure on the Web where visual appearance is subject to (ever-changing) fashion, trends, and new technical features [29]. In addition, as application designers cannot expect Web users to consult a user's manual Web applications need to be selfexplanatory requiring particular attention to visual design and the consistency of the interaction style behaviour.

\subsection{Usage-Related Characteristics}

Unlike in more traditional settings, the users of Web applications often vary in numbers and cultural background, use heterogeneous devices and can freely choose the time and location of accessing the Web application [18]. Developers frequently cannot predict all these potential settings. 
Natural context. This includes aspects of the location and time of access, offering the opportunity of new kinds of context-based services, not least due to the advent of mobile computing. In addition, the possibility of immediate and permanent availability of Web applications requires special quality considerations such as 24/7 availability.

Unpredictable technical infrastructure. Available end-user devices vary in hardware and software capabilities such as display size, computational power, or browser version. Also network connections differ with respect to bandwidth, reliability, stability and availability, all affecting the quality of service [29]. Complexity is increased even further due to the fact that the actual representation of the Web application on the client device is to a large extent outside the control of the developers. For example, users configure their browsers individually and may even disallow certain essential features (e.g., cookies or JavaScript).

Diversity and magnitude of user base. Web application users differ in age, social and cultural backgrounds, goals, intentions, skills, and capabilities [17]. These heterogeneity has to be considered by application developers since the Web entails no obligation and Web applications will only be used if they bring immediate advantage. The way users interact with the Web application can be hardly predicted and users may leave the Web application at any time [15]. Also, the number of users accessing the Web application may vary considerably making scalability another crucial quality aspect.

\subsection{Development-Related Characteristics}

Web application developers need to deal with conditions, risks, and uncertainties not always present in traditional software projects.

Development team. Web application development is a multi-disciplinary effort comprising a mixture of print publishers, authors, software developers, marketing experts, and art designers [26]. Such teams are also dominated by significantly younger team members which are less willing to adhere to conventions and more inclined towards applying new (and often still immature) technologies [24]. Another important characteristic is the involvement of open source communities.

Development environment. The technical infrastructure used for developing a Web application is characterized by a high degree of volatility and heterogeneity. Web application development relies on a broad spectrum of different COTS components (e.g., Web server, application server, database system, publishing framework etc.). Because of the increased time-to-market pressure these components are often immature and fall short in stability, reliability, and desired functionality.

Legacy integration. Web applications often need to integrate legacy systems [21]. The external services provided by these systems are, however, rarely documented and often change without notice, thus negatively affecting the quality of the overall Web application.

Process. Web application development processes are characterized by frequent changes and adjustments, which are necessary due to rapid technological develop- 
ments, fast changing trends, volatile requirements, and rigid schedules. This calls for highly iterative, flexible, and prototype-oriented development methods [3, 24].

\subsection{Evolution-Related Characteristics}

Web applications are subject to frequent changes and permanent evolution. Their development is driven by rapidly changing technology and the volatility of Web users leads to a highly competitive situation where immediate Web presence and short time to market are considered crucial: "Unlike conventional application software that evolves over a series of planned, chronologically spaced releases, Web applications evolve continuously." [28]. In the course of evolution, negotiability of quality often sacrifices maintainability $[14,20]$.

\section{Conclusion}

To say it with the words of Robert Glass [13], “... there have always been important differences between software projects, diversity is the key part of software development. Information systems have always been developed differently from scientific systems, and critical projects have been treated differently from more mundane ones. Why should we be surprised that the same condition holds for Web and non-Web projects?" There are, however, important differences between the two, urgently requiring to systematically re-think the applicability and appropriateness of existing solutions in the whole area of software engineering, eventually based on the knowledge areas defined by the SWEBOK [4]. The clarification of this issue would be a major step towards establishing Web Engineering as a discipline on its own.

\section{References}

[1] Balasubramaniam, R., Pries-Heje, J., Baskerville, R., Internet Software Engineering: A Different Class of Processes, Annals of Software Engineering, 14 (1-4), Dec., 2002, pp. 169-195.

[2] Berners-Lee, T., WWW: Past, Present, and Future, IEEE Computer, 29 (10), Oct., 1996, pp. 69-77.

[3] Boehm, B., Turner, R., Balancing Agility and Discipline, A Guide for the Perplexed, Pearson Education, 2004.

[4] Bourque, P., Dupuis, R., Abran, A., Moore, J. W., Tripp, L. L. The Guide to the Software Engineering Body of Knowledge, IEEE Software, vol. 16, pp. 35-44, Nov/Dec. 1999.

[5] Botterweck, G. and Swatman, P. A., Towards a Contingency Based Approach to Web Engineering. 7th Australian Workshop on Requirements Engineering (AWRE), Melbourne, 2002. 
[6] Conklin, J., Hypertext: An Introduction and Survey, IEEE Computer, 20 (9), Sept., 1987, pp. 17-41.

[7] Cutter Consortium, Poor Project Management Number-one Problem of Outsourced Eprojects, Cutter Research Briefs, November, 2000, http://www.cutter.com/research/2000/ crb001107.html.

[8] Deshpande, Y., Hansen, S., Web Engineering: Creating a Discipline among Disciplines, Special Issue on Web Engineering, IEEE Multimedia, 8 (2), April-June, 2001, pp. 82-87.

[9] Deshpande, Y., Hansen, S., Murugesan, S., Web Engineering: Beyond CS, IS and SE An Evolutionary and Non-Engineering View, Proc. of the $1^{\text {st }}$ ICSE Workshop on Web Engineering (held in conjunction with the Int. Conference on Software Engineering (ICSE), Los Angeles, May, 1999.

[10] German, D., Cowan, D., Towards a Unified Catalogue of Hypermedia Design Patterns, Proc. of the $33^{\text {rd }}$ Hawaii International Conference on System Sciences (HICSS), Maui, Hawaii, January, 2000.

[11] Ginige, A., Web Engineering in Action, Proc. of the $2^{\text {nd }}$ ICSE Workshop on Web Engineering (held in conjunction with the Int. Conf. on Software Engineering (ICSE), Limerick, Ireland, June, 2000.

[12] Glass, R.L., Searching for the holy grail of software engineering, Communications of the ACM 45(5), 2002.

[13] Glass, R.L., A mugwump's-eye view of Web work. Communication of the ACM 46(8), 2003.

[14] Grünbacher, P., Ramler, R., Retschitzegger, W., Schwinger, W., Making Quality a FirstClass Citizen in Web Engineering, Proc. of the 2nd Workshop on Software Quality (SQW), held in conjunction with the 26th Int. Conf. on Software Engineering (ICSE), Edinburgh, Scotland, May, 2004.

[15] Holck, J., Clemmensen, T., What Makes Web Development Different?, Presented at the 24th Information Systems Research Seminar in Scandinavia (IRIS) Ulvik, Norway, August 2001.

[16] Kautz, K. \& J. Nørbjerg: Persistent Problems in Information Systems Development: The Case of the World Wide Web, in Ciborra et. al. (eds.): Proc. of the European Conf. on Information Systems, Naples, Italy, June, 2003

[17] Kobsa, A., Generic User Modeling Systems, User Modeling and User-Adapted Interaction, 11 (1-2), Ten Year Anniversary Issue, 2001, pp. 49-63.

[18] Kappel, G., Pröll, B., Retschitzegger, W., Schwinger, W., Customisation for Ubiquitous Web Applications - A Comparison of Approaches, International Journal of Web Engineering and Technology (IJWET), Inderscience Publishers, January, 2003.

[19] Kappel, G., Pröll, B., Reich, S., Retschitzegger, W., (eds.), Web Engineering - Systematic Development of Web Applications, dpunkt.verlag, 2004.

[20] Levine, L., Baskerville, R., Loveland Link, J.L., Pries-Heje, J., Ramesh, B., \& Slaughter, S.. Discovery colloquium: Quality software development @ Internet speed. (SEI Technical Report CMU/SEI-2002-TR-020) Pittsburgh, Software Engineering Institute, 2002.

[21] Lowe, D., Hall, W., Hypermedia and Web: An Engineering Approach, Wiley \& Sons, 1999.

[22] Lowe, D., Engineering the Web - Web Engineering or Web Gardening? WebNet Journal, 1 (1), January-March, 1999.

[23] Lowe, D., Web System Requirements: An Overview, Requirements Engineering Journal, Vol. 8, No. 2, 2003 
[24] McDonald, A., Welland, R., Web Engineering in Practice, Proc. of the $4^{\text {th }}$ Workshop on Web Engineering (in conjunction with $10^{\text {th }}$ Int. Conf. on WWW), Hong Kong, Mai, 2001.

[25] Murugesan, S., Deshpande, Y., Hansen, S., Ginige, A., Web Engineering: A New Discipline for Web-Based System Development, Proc. of the $1^{\text {st }}$ ICSE Workshop on Web Engineering (held in conjunction with the Int. Conference on Software Engineering, ICSE), Los Angeles, USA, May, 1999.

[26] Powell, T., Jones, D., Cutts, D., Web Site Engineering: Beyond Web Page Design, Prentice Hall, 1998.

[27] Pressman, R. S., Can Internet-Based Applications Be Engineered?, IEEE Software, 15 (5), September-October, 1998, pp. 104-110.

[28] Pressman, R. S., What a Tangled Web We Weave, IEEE Software, 17 (1), Jan/Feb., 2000, pp. 18-21.

[29] Pressman, R. S., Chapter 29: Web Engineering. In: Software Engineering: A Practitioner's Approach, 5. Edition, McGraw-Hill, 2000.

[30] Strong, D.M., Lee, Y.W., Wang, R.Y., Data Quality in Context, Communications of the ACM, 1997. 40(5): pp. 103-110.

[31] Taylor, M.J., McWilliam, J., Forsyth, H., Wade, S., Methodologies and website development: a survey of practice, Information and Software Technology, 44, 2002.

[32] Vidgen, R., What's so different about developing web-based information systems? Proc. of 10th European Conference on Information Systems, Gdansk, Poland, June 2002. 